\title{
Berkeley Accelerator Space Effects (BASE) Light Ion Facility Upgrade
}

\author{
M.B. Johnson, M.A. McMahan, T.L. Gimpel, W.S. Tiffany
}

\begin{abstract}
The BASE Light Ion Facility upgrades have been completed. All proton beams are now delivered to Cave 4A. New control software, a larger diameter beam window, and improved quality assurance measures have been added.
\end{abstract}

Index Terms-BASE Facility, Cyclotron, Protons, Radiation Effects Testing

\section{INTRODUCTION}

$\mathrm{T}_{\mathrm{D}}^{\mathrm{H}}$ HE 88-Inch Cyclotron, part of the Nuclear Science Division at Lawrence Berkeley National Laboratory, is the home of the Berkeley Accelerator Space Effects (BASE) Facility [1] and supports a local research program in nuclear science. A facility of the Department of Energy's (DOE) Office of Science, the Cyclotron is jointly funded by the DOE and the National Security Space Community. The BASE Facility provides well-characterized beams of protons, heavy ions and other medium energy particles that simulate the space environment.

Previously, all proton runs were completed in Cave 3 and all heavy ions in Cave 4B. For customers requiring both protons and heavy ions, this arrangement necessitated moving test equipment between the two locations. This major inconvenience has been mitigated by moving the entire Light Ion Facility to Cave 4A (Fig. 1), immediately adjacent to the Heavy Ion Facility. At the same time, the maximum beam size was increased to $15 \mathrm{~cm}$ and both the hardware and software was upgraded.

M. B. Johnson, M.A. McMahan, T.L. Gimpel, and W.S. Tiffany are with the Lawrence Berkeley National Laboratory's 88-Inch Cyclotron BASE Facility, Berkeley, CA 94720 USA (phone: 510-486-4389; fax: 510-486-6122; e-mail: mbjohnson@ boulder.nist.gov).

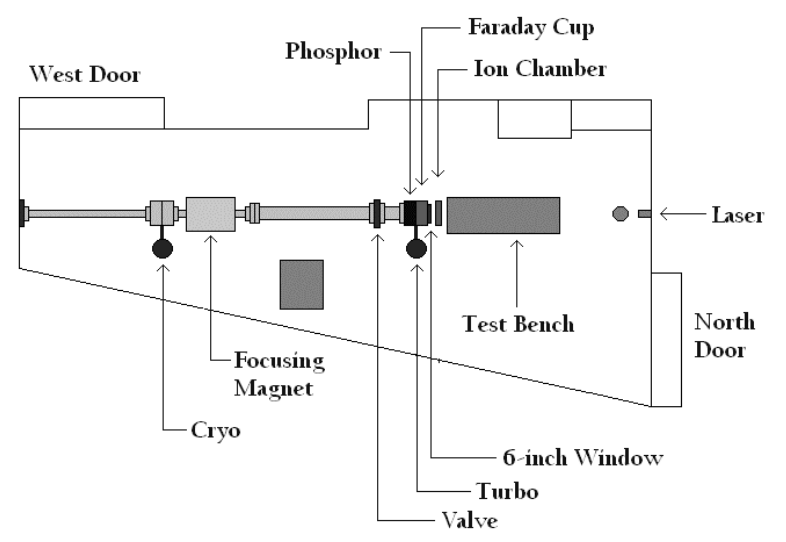

Fig. 1. Light Ion Facility in Cave 4A

\section{METHODS AND CAPABILITIES}

\section{A. Standard Configuration}

Under standard operating conditions, using a positionsensitive ion chamber for online dosimetry, the BASE Light Ion Facility is capable of providing flux densities from $1 \times 10^{3}$ to $1 \times 10^{10}$ protons $/ \mathrm{cm}^{2}$-sec at beam diameters of up to $15 \mathrm{~cm}$. Proton testing is normally performed in air. Standard proton energies include 13.5, 20, 30, 40, 50, and $55 \mathrm{MeV}$, though energies between these values are also available. All energy changes are accomplished by performing a 1-hour retune of the Cyclotron, enabling precise energy values to be obtained.

To tune the beam into the cave, protons created at our ion source are accelerated through the Cyclotron and sent down the beamline. The beam is then spread out evenly on the cave phosphor with focusing magnets and foils by the Control Room Operator. Beam efficiency from the beam stop at the exit of the Cyclotron to the Faraday cup in Cave 4A, with a fully-spread beam, is approximately $30 \%$. The beam exits the vacuum envelope through an innovative new $15-\mathrm{cm}$ diameter thin-film window [2], constructed of 5-mil Kapton (Fig. 2). This new window, which allows for testing larger quantities or sizes of parts compared to our previous window, is a significant improvement for many of our customers desiring greater exposure area. 


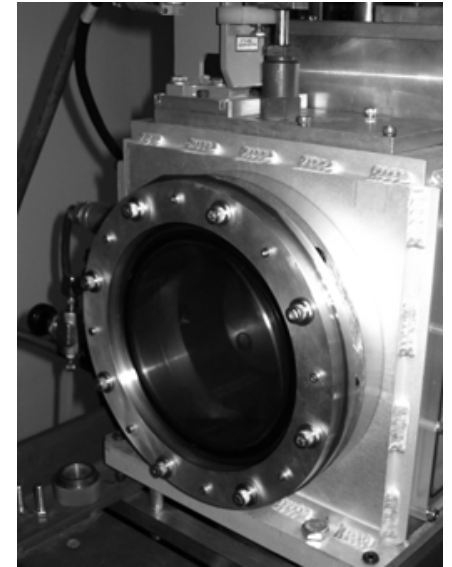

Fig. 2. Beam Exit Window

The ion chamber (Fig. 3) monitors the beam continuously during the experiment. Beam particles traveling through the nitrogen-filled ion chamber leave a trail of ions that are collected by gold-coated foil electrodes, the resulting current of which is measured with the electronics. The current signal from the ion chamber is routed through a current to frequency converter, where the pulses are then counted with a CAMAC scaler module and sent to the computer. The computer is located in the BASE test shack directly above Cave 4A.

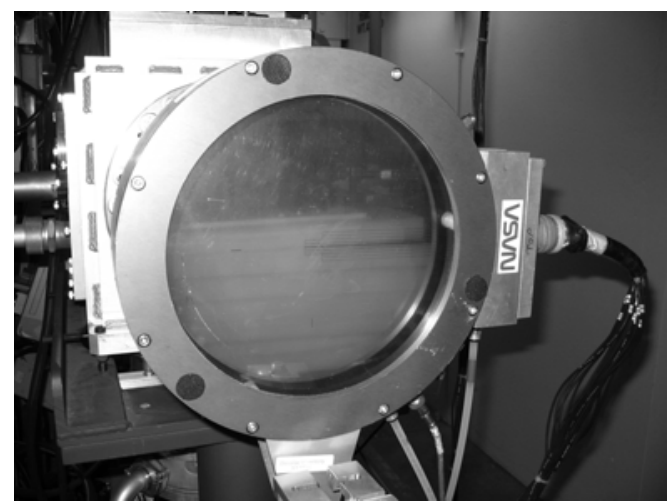

Fig. 3. Ion Chamber

The final processing and display is performed with recently upgraded LabView software (Fig. 4), which consolidated previous versions and provide an intuitive interface for the customer.

The current from the ion chamber's six concentric rings (with diameters of 1, 2, 4, 6, 8, and $10 \mathrm{~cm}$ ) and four quadrants are displayed by the software, and used to establish and monitor uniformity. For a typical run, the concentric rings and quadrants are kept within 5\% of each other. Any change in beam uniformity or position due to magnet drift is easily identified by monitoring the ion chamber data.

The customer can choose to control and monitor the beam by fluence and silicon dose (for chip testers), or by water dose (for biology experiments). Fluence or dose limits can be set that will stop the beam at the ion source upon reaching the desired

level. Flux density is continuously plotted in real time. New logging capabilities have been added to automatically record run data, which is stored on the computer desktop and easily retrievable by the user.

\section{B. Quality Assurance}

As a quality assurance measure to verify ion chamber performance, we recently developed software (Fig. 5) that analyzes the Gafchromic RTQA proton films taken at the beginning of each run. First, the films are scanned in to a desktop computer as a grayscale image, where each pixel is assigned an integer value between 0 and 255. Then, using our new "Filmalyzer" software developed by Cyclotron staff, the boundaries and shape (square or round) of the region of interest are selected by the experimenter. Based on the desired resolution, the image is automatically sorted into a calculated number of 400-pixel (20x20) blocks. Each block is then summed and divided by 400 . This process creates manageable arrays of sufficient resolution, while reducing noise due to dust, film blemishes, and faulty pixels. Background points are selected to serve as a reference, and a "percent flatness" is determined. Maximum and minimum exposure points are displayed in both a three-dimensional graph and a profile plot, with typical flatness values of $+/-5 \%$ for an $8 \mathrm{~cm}$ beam diameter (Table 1). This data is now printed and provided to the customer at the beginning of each run.

Table 1. Percent Flatness Results

\begin{tabular}{|c|c|c|c|}
\hline Date & Proton Energy (MeV) & \%Flatness $(+)$ & \%Flatness(- \\
\hline$\overline{3 / 13} / 06$ & 50 & 2.62 & 4.24 \\
\hline $5 / 22 / 06$ & 50 & 4.25 & 4.97 \\
\hline 6/9/06 & 50 & 3.08 & 2.62 \\
\hline 6/19/06 & 50 & 2.76 & 1.88 \\
\hline
\end{tabular}

Additional testing was performed to determine the correct exposure point for the film. Film samples $(2.5 \mathrm{~cm}$ diameter $)$ were exposed to $50 \mathrm{MeV}$ protons in $25 \mathrm{Rad}$ increments, from 25 to 300 Rads, as measured by the ion chamber. The samples were then analyzed with the "Filmalyzer" software to determine the mean (film) pixel exposure. Results from this analysis (Fig. 6) indicate that doses of 75 to 100 Rads provide relatively linear exposure characteristics and are well-suited for verifying ion chamber performance. Future work will investigate differences in film exposure to varying proton energies. 


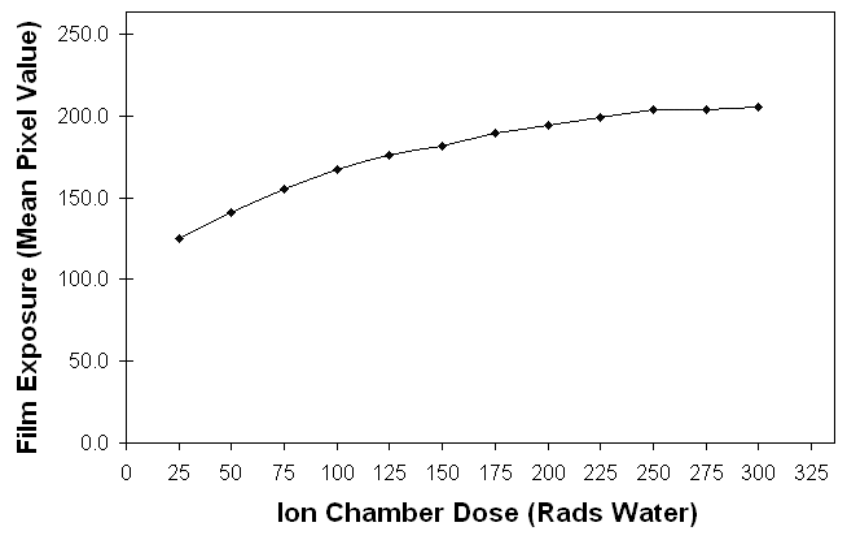

Fig. 6. Ion Chamber Dose vs. Film Exposure

\section{Non-standard Configurations}

Energies from 2 to $13.5 \mathrm{MeV}$ can be obtained by using the vacuum chamber and heavy ion dosimetry in the adjacent Cave $4 \mathrm{~B}$, or by removing the ion chamber and measuring dose on a Faraday cup. Lower flux densities are accommodated by using a scintillator in place of the ion chamber.

Higher flux densities, between $1 \times 10^{10}$ and $5 \times 10^{11}$ protons $/ \mathrm{cm}^{2}$-sec, require ion chamber removal and Faraday cup monitoring either intermittently upstream or continuously downstream of the part being tested. Additional safety requirements will be necessary for these configurations due to the potential for contamination from target and beam stop materials and high activation.

\section{Future Upgrades}

We are currently investigating new shielding and beamline configuration changes which will allow operating at higher flux densities with minimal additional training. We also plan to install a set of proton beam degraders which will enable faster energy changes. Ion chamber calibration is being reviewed, and we are designing a procedure which will conform to the industry standards presently under discussion [3].

\section{E. Additional Information}

Additional training may be required to run with protons at flux densities of greater than $1 \times 10^{8}$ protons $/ \mathrm{cm}^{2}$-sec. Shielding materials, laser alignment tools, and mounting fixtures are available. Holes (10 $\mathrm{cm}$ in diameter) are provided through the cave shielding blocks for connecting additional test equipment, with a distance of approximately 10 feet from the test bench to the top of the shielding block (one-third of our previous length for Cave 3). A total of 10 BNC cables and 4 SHV cables are permanently installed and available for use; additional cables are easily added by the customer. Collimators constructed of $1.25-\mathrm{cm}$ thick aluminum are now available with diameters of 2.5, 5, 7.5, and $10 \mathrm{~cm}$, and mount between the ion chamber and the device under test.
Parts activated with protons often take days or weeks to decay to background levels. These parts can be released by either waiting for the total activity to decay to background, or preferably by sending us a "rad license" with the required isotope and activity limits. Gamma spectroscopy can be performed at the BASE Facility upon request. Cyclotron and Berkeley Lab staff are available to help customers navigate through the process.

\section{ACKNOWLEDGMENT}

This work was supported by the U.S. Department of Energy under contract No. DE-AC02-05CH11231 and NNSA Grant No. NA00078.

\section{REFERENCES}

[1] M.A. McMahan, The Berkeley Accelerator Space Effects (BASE) Facility, Proceedings of the Nuclear Space Conference 2005, San Diego, CA, USA.

[2] J.L. Western, Mechanical Safety Subcommittee Guideline for Design of Thin Windows for Vacuum Vessels (FERMILAB-TM-1380), Fermi Ntnl. Accelerator Laboratory, Batavia, IL, USA.

[3] ICRU Report 59, Clinical proton dosimetry Part 1: Beam production, beam delivery and measurement of absorbed dose, International Commission on Radiation and Units, Bethesda, MD, USA. 


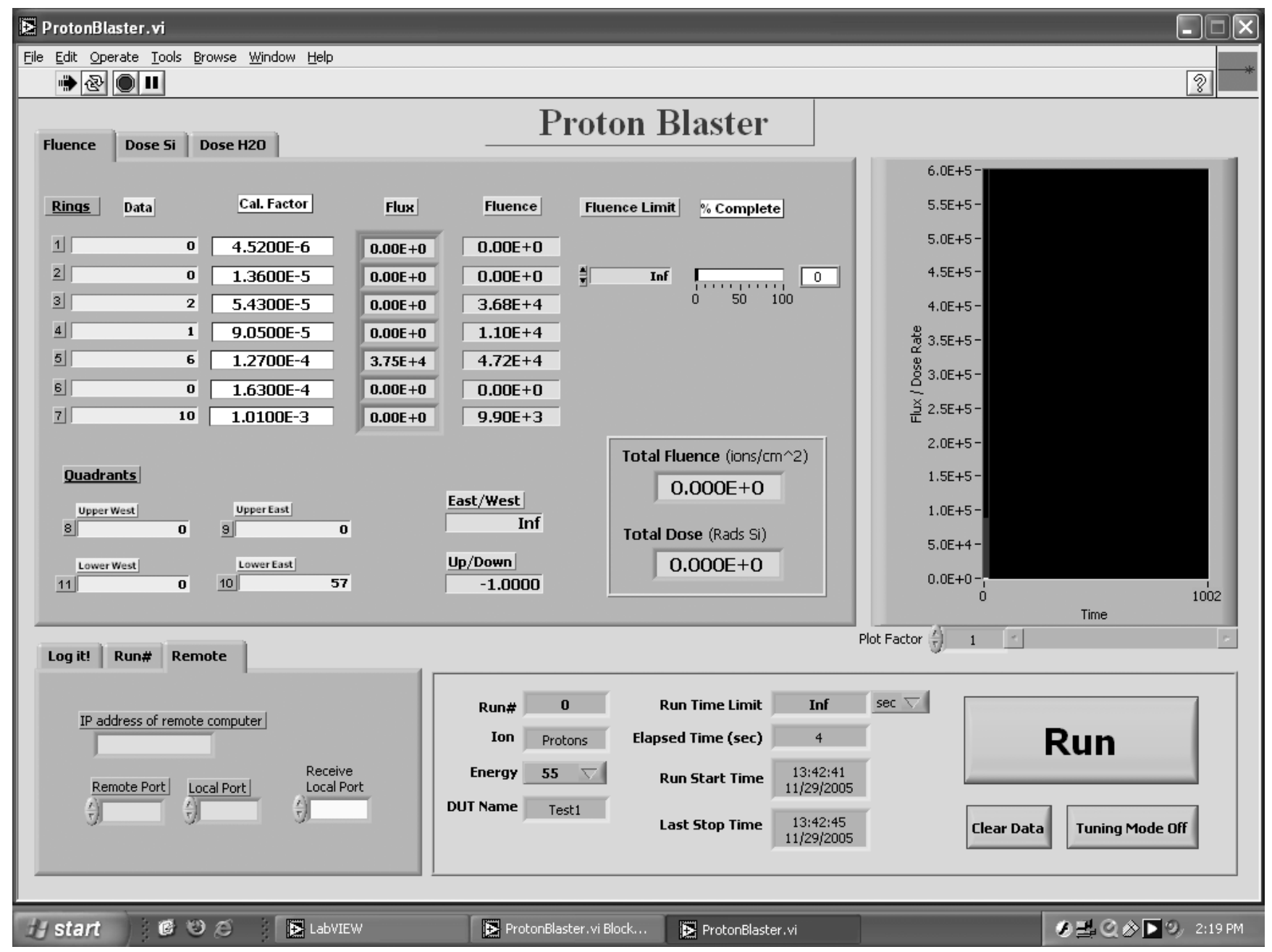

Fig. 4. Control Software 


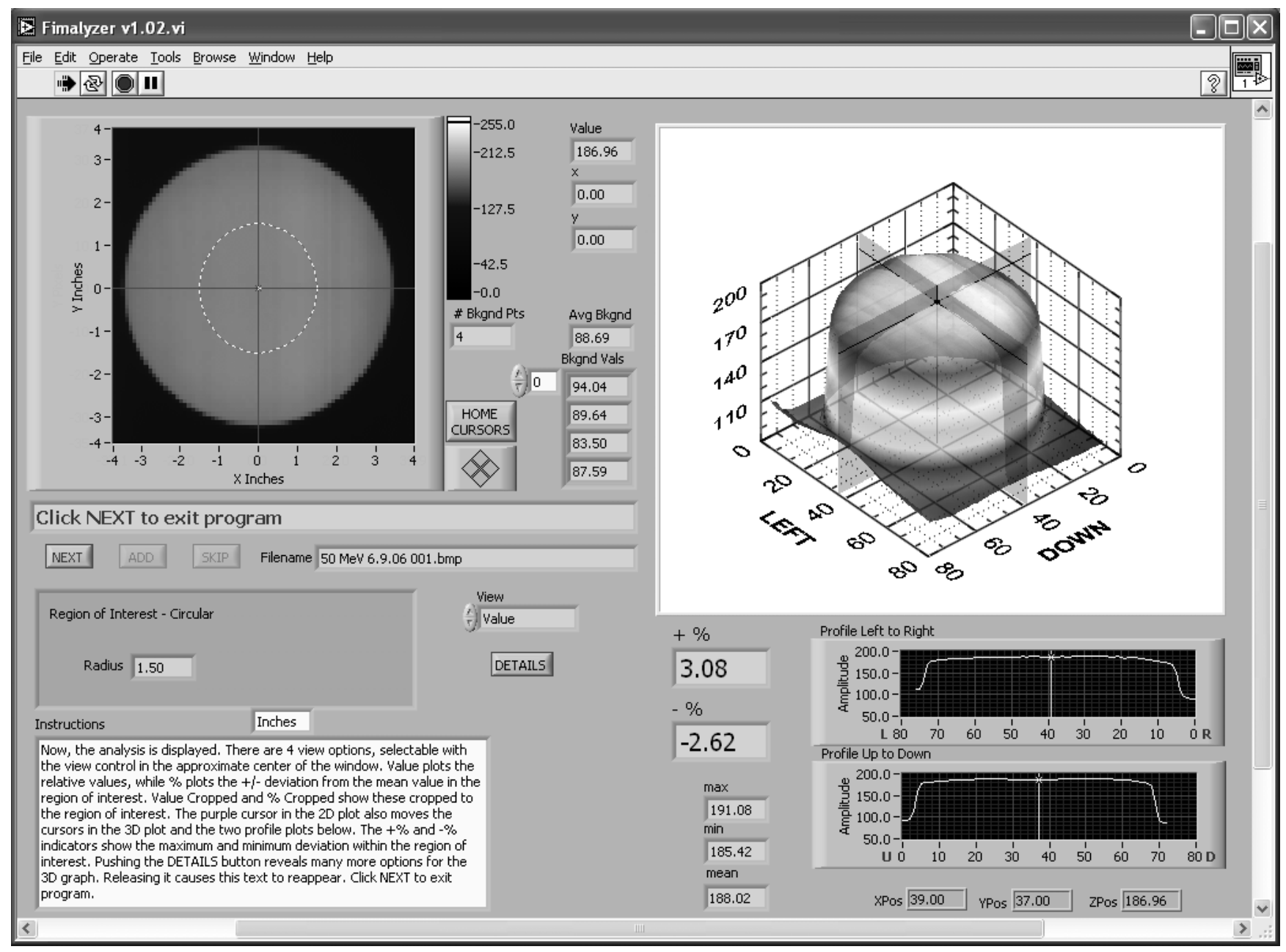

Fig. 5. "Filmalyzer” Analyzing Software 